Décadrages Décadrages

cinéma, à travers champs Cinéma, à travers champs

32-33 | 2016

Séries télévisées contemporaines

\title{
Entretien avec Kim Seob Boninsegni
}

\section{Charlotte Bouchez et Tristan Lavoyer}

\section{(2) OpenEdition}

\section{Journals}

Édition électronique

URL : http://journals.openedition.org/decadrages/954

DOI : $10.4000 /$ decadrages. 954

ISSN : 2297-5977

\section{Éditeur}

Association Décadrages

\section{Édition imprimée}

Date de publication : 1 juillet 2016

Pagination : 182-190

ISBN : 978-2-9700963-2-0

ISSN : 2235-7823

Référence électronique

Charlotte Bouchez et Tristan Lavoyer, «Entretien avec Kim Seob Boninsegni », Décadrages [En ligne], 32-33 | 2016, mis en ligne le 01 décembre 2018, consulté le 19 avril 2019. URL : http:// journals.openedition.org/decadrages/954; DOI : 10.4000/decadrages.954

® Décadrages 


\title{
Entretien avec Kim Seob Boninsegni
}

\begin{abstract}
Kim Seob Boninsegni vit et travaille à Genève. Son travail entrecroise différentes pratiques artistiques, et nous l'avons rencontré dans son atelier pour discuter avec lui de son choix d'investir davantage la production cinématographique avec son film Occupy the Pool (2015). Dans cet entretien, nous avons cherché à dégager les spécificités de son approche du cinéma, basée sur une position initiale d'extériorité par rapport à ce domaine. Notre démarche a consisté à questionner l'impact des conditions de production tant sur le film lui-même que sur des aspects plus informels qui constituent la genèse d'un tel objet.
\end{abstract}

Décadrages - Peux-tu nous décrire le parcours qui t'a amené à t'intéresser plus spécifiquement au film?

Kim Seob Boninsegni - Mon parcours est un peu étrange. S’il est redevable à beaucoup de disciplines, c'est avant tout l'écriture qui m’a poussé à entrer à la HEAD en cinéma en 1997. A cette époque, la vidéo était le médium roi et en parallèle j’avais un travail alimentaire au Centre pour l'Image Contemporaine de Genève. Une des tâches qui m’avait été confiée consistait à visionner les vidéos de leur médiathèque pour en relever la durée et quelques autres critères. C'est à ce moment que j’ai eu mes premiers contacts avec la vidéo d'art et son univers souvent étrange. Cela a probablement eu une incidence sur mon parcours, puisque à la fin de la première année en cinéma je quittais cette section pour celle des arts visuels. J'y rencontrais Dominique Gonzalez-Foerster qui fut ma professeure à Genève puis au Palais de Tokyo à Paris et qui contribua à enrichir ma vision de ce monde interstitiel entre cinéma et art contemporain.
A la suite de cette période, l'intérêt général pour les nouvelles technologies et la vidéo d'art déclina et ma pratique devint plus plasticienne jusqu'en 20 Io. A partir de ce moment, on me proposa de créer un spectacle avec Marie-Caroline Hominal au Théâtre de l'Usine et cette nouvelle expérience me fit renouer avec la mise en scène. Deux autres spectacles suivirent, une performance et une chorégraphie, et mon regain d'intérêt pour le cinéma se concrétisa par ce projet de film.

As-tu écrit un scénario pour le film? Ou te basais-tu davantage sur le canevas à partir duquel tu laissais une place à l'improvisation?

L'accès au cinéma (surtout de fiction) pour les artistes dits «plasticiens» est complexe, voire par certains aspects absurde. J'en discutais dernièrement avec une artiste embarquée elle aussi dans un projet long. Pour présenter nos projets auprès de commissions afin d'obtenir des financements, nous devons très souvent 
nous baser sur des critères très contraignants comme l'écriture d'un scénario classique pour ensuite le déconstruire lors du tournage puisque ce genre de document est souvent peu adapté pour les projets venant des plasticiens.

A la base d'Occupy the Pool, il y a effectivement eu une écriture et un scénario relativement classiques, coécrit avec Marianne Thivillier. En fait, le premier tournage fut un échec pour de nombreuses raisons, à commencer par une météo défavorable. Le jeu des interprètes posait aussi souci puisqu'il y avait une disparité énorme entre les types de jeux de chacun, et ce malgré les répétitions et tests préalables. A ce moment-là, j’ai décidé de réécrire le scénario dans un style qui me soit plus naturel. Je ne sais pas s'il est redevable de l'art contemporain, mais j'ai opté pour une immersion accrue dans l'univers des interprètes, un peu comme pour les spectacles que je mettais en scène en parallèle.

La seconde version du scénario se base donc sur cette approche immersive et offre ce point de vue intérieur au groupe filmé. C'est une écriture qui compile des saynètes ou des micro-performances, et compense le manque de continuité dans le récit par... disons un système «bressonien», où les interprètes ne cessent de se passer les mêmes objets durant tout le film. Cela caractérise assez bien notre rapport à l'improvisation pour ce film. Si la structure était effectivement écrite avec des directions de dialogues, une trame, un début et une fin, il était difficile de prévoir concrètement quels types d'images et de sons nous obtiendrions au final. Une anecdote à ce propos: si les interprètes ne cessent de chercher du feu dans ce film, c'est parce que au début de chaque journée de tournage nous leur confisquions leurs briquets. Ce sont ce type de petites procédures qui ont beaucoup contribué à une improvisation relativement maîtrisée.
Cette méthode relève-t-elle de théories anthropologiques ou sociologiques?

Je dois avouer que j’apprécie énormément les écrits de Bruno Latour, mais je ne suis pas certain que cela atteste d'un point de vue sociologique plutôt qu'anthropologique. Plus simplement, je pense que cette approche est liée à ma pratique de l'art contemporain, mais aussi à ma biographie (je suis adopté). C’est une

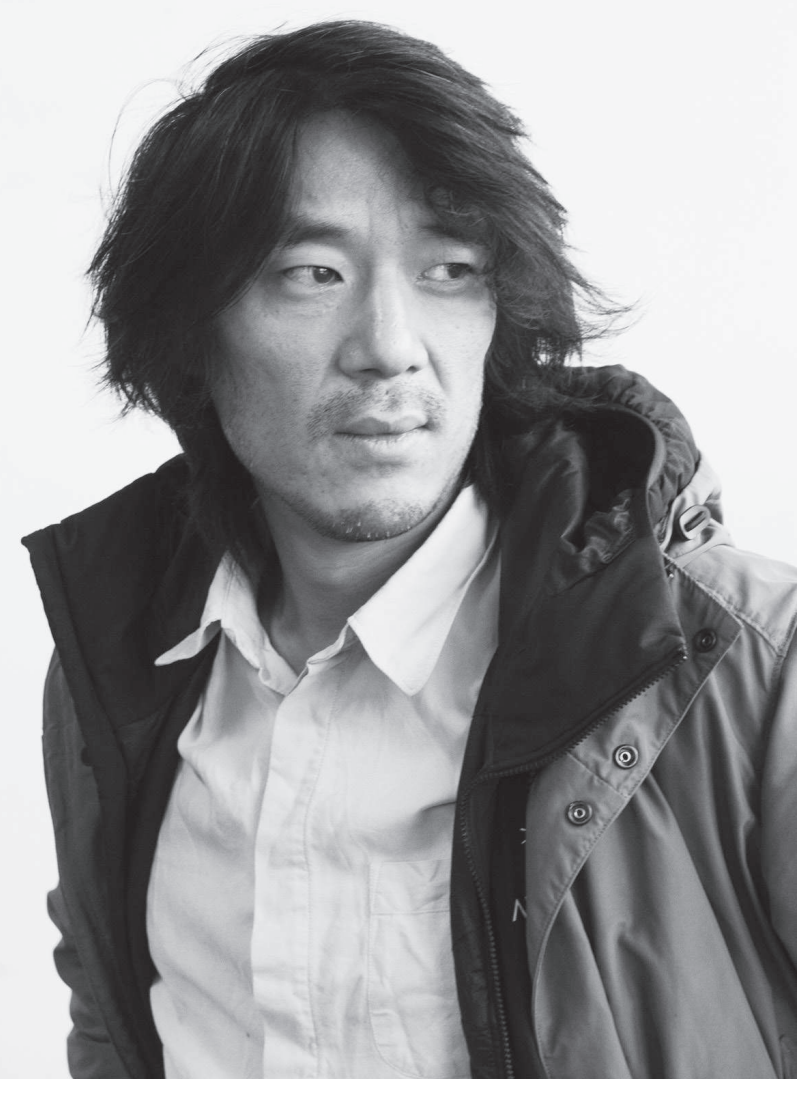

(c) Yvan Alvarez 2015 
approche qui ressemble beaucoup à celle des flics sous couverture. D'une certaine manière, c'est se faire adopter par son sujet.

Les acteurs avaient-ils une marge d'improvisation, et si oui, comment cela se passait-il lors du tournage?

Tout s'est passé au niveau de l'écriture. Si la première version était un scénario, la seconde a davantage emprunté au déroulé. En effet, à part deux scènes complètement improvisées, tout le reste est écrit. Pour moi, il s'agissait de placer le processus de décision en amont; c'est-à-dire de définir une situation qui opérerait sur le jeu des interprètes plutôt qu'une direction forte lors du tournage. Cela demande de faire particulièrement confiance à l'image (et à Gabriel Lobos, le chef opérateur).

Est-ce que tu as discuté avec les interprètes du rôle qu'ils souhaitaient jouer?
Non. Ou plutôt si, mais il faut avoir conscience que ce type de film n'est pas familier des interprètes et de leur définition de ce que sont le cinéma et le film. On en revient à des questions de méthode et d'approche. Comme pour les spectacles - les interprètes d'Occupy the Pool sont les mêmes que pour la plupart des spectacles mis en scène durant la même période - mon approche consistait à beaucoup observer et interroger. Par exemple, le film contient une scène où Timothée Calame fait boire Camille. C'est une image que j'ai vue pendant un spectacle alors qu'ils se préparaient dans les coulisses. Camille était dans un costume ridicule qui lui ôtait l'usage de ses bras et c'est Tim qui délicatement la faisait boire. C’est une image forte qui m'est restée et s'est retrouvée dans le film.

Cette méthode prend énormément de temps, c'est d'ailleurs en partie pour cela que le premier tournage a échoué, car je n'avais que six mois de connaissance des interprètes. Au final, l'écriture ici ne crée pas

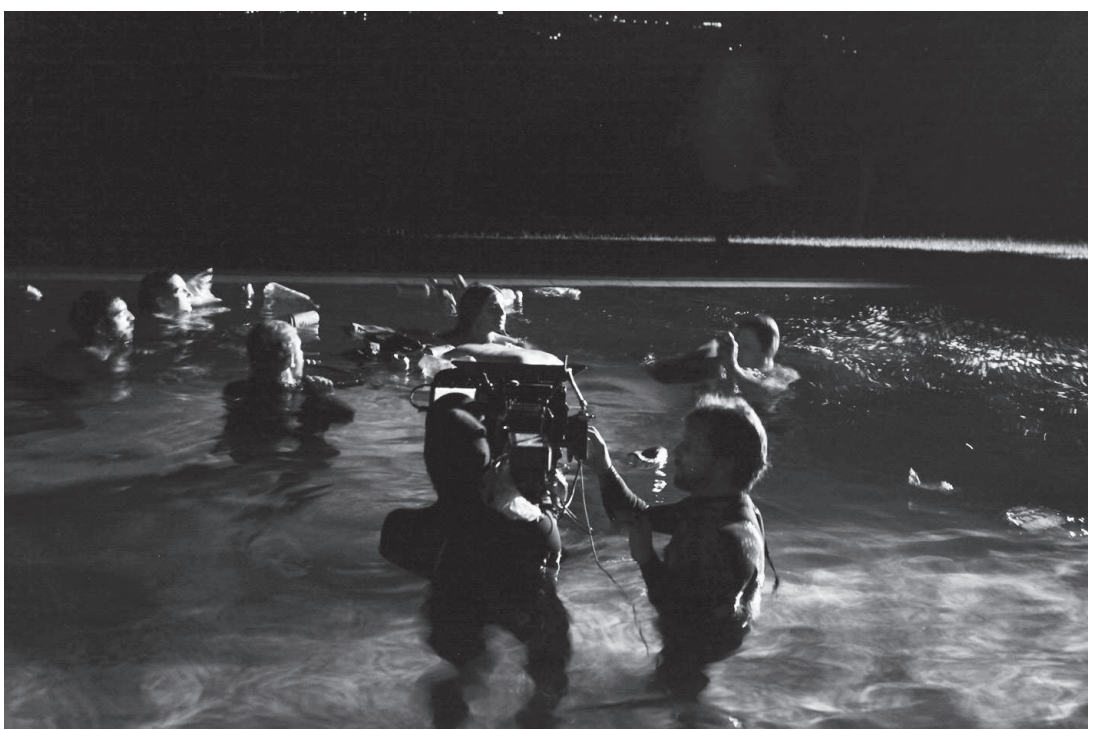


réellement de personnages. Elle reprend certaines caractéristiques réelles des interprètes et les adapte à la trame désirée. Mélanie a certainement chipé des clopes durant ses soirées, mais je doute qu’elle ait été jusqu'à les entasser dans son sac. C'est là qu'intervient l'écriture, en activant une possibilité donnée par cette personne. Cela a pu être compliqué pour eux, car cela revenait à interpréter une version décalée d'eux-mêmes.

Tu parles d'une continuité logique entre les spectacles et le film. Pourrais-tu préciser en quoi ton activité théâtrale imprègne-t-elle ta pratique cinématographique; s'agit-il d'une sorte de transfert d'expérience?

Le théâtre (en fait plutôt la performance et la chorégraphie) constitue déjà une sorte de transfert ou de traduction de mon expérience de l'art contemporain. C'est d'ailleurs ainsi que le monde de la scène recycle parfois les plasticiens, par le biais scénographique ou spatial. Pour Occupy the Pool il y a effectivement un apport de cette expérience puisque la partie «piscine» est appréhendée comme une scène avec un côté cour et jardin, et un bord servant à la prise de vue. Lautre aspect est plus spécifique à la danse, puisque cela m’a apporté une sensibilité quant au corps des interprètes. Dans Occupy the Pool il y a d'ailleurs peu de dialogues, mais beaucoup de plans présentant leurs corps et leurs mains, avant que cela ne disparaisse dans la piscine.

Tu parles d'anatomie. Mais tu procèdes presque à une construction d'un «corps-objet»?

Oui. A l'instar des objets qui se baladent. D'une certaine manière, ils sont sur le même plan. Non pas que ce groupe ou cette génération soit matérialiste ou consumériste, mais plutôt parce que, si notre génération a grandi dans des friches postindustrielles, eux ont plutôt grandi dans les coulisses des centres commerciaux. En atteste leur attrait pour les arcades commerciales ou les «malls» lorsqu'ils ouvrent des espaces d'art. Il y a donc quelque chose de cohérent à les placer sur un plan analogue. Un peu comme si une génération se voyait jouer avec les rebus d'une société de consommation procédant de leurs parents. Et puis il y a le personnage de Camille. C'est sur elle que s'inscrit le titre du film, c'est elle aussi qui a la seule scène un peu chaude du film, et c'est aussi elle qui passe d'un membre du groupe à l'autre alors qu'elle n'a quasiment aucune ligne de dialogue.

On pourrait dire que tu utilises les caractéristiques corporelles pour construire le récit. II ne s'agit peut-être pas tant du corps-objet que l'on conditionne ou manipule que d'un corps comme construction d'un langage.

Oui, ou d'un corps curseur. Un peu comme les suivis très longs de personnages dans le cinéma asiatique des années 1990. Un corps assez anonyme que l'on suit, avec lequel on déambule de manière nonchalante et par lequel se dévoile en fond le réel comme décor. C'est comme dans l'ouverture de Millenium Mambo (Hou Hsiao-Hsien, 20oI) par exemple, ou dans la manière dont les corps sont traités dans The River (I997) de Tsai Ming-liang, qui m’a énormément marqué.

\section{Le dialogue est donc très peu écrit?}

C'est un peu comme un parfum. Une direction. On tend à cela, mais on ne sait pas réellement ce que cela va donner. Je leur ai beaucoup demandé de panacher, c'est-à-dire de prendre en compte les coordonnées que je leur donnais, par exemple de mentionner un personnage fictif à intégrer dans une discussion qu’ils auraient pu avoir sans caméra.

Attribues-tu un enjeu à la situation? 
Pour ce projet, j’ai appris à ne pas avoir trop d'attentes précises; ou disons que la précision se situait à un autre niveau. Tout le «récit» dépendait de leur manière de savoir jouer en partie leur propre rôle et celui qui leur était demandé en plus de la gestion des situations de tournage parfois compliquées: comme cette nuit dans la piscine à $9^{\circ}$ où Marion Poisot a réellement souffert. Cet aspect de la direction n'a d'ailleurs pas complètement fonctionné, car cela dépendait beaucoup des personnes choisies. Avec Sabrina Röthlisberger, Gaïa Vincensini, Alan Schmalz ou Mélanie Veuillet cela fonctionnait bien, il suffisait de leur donner une indication et ils s'amusaient énormément avec. D’autres interprètes étaient vraiment tétanisés, car ils devaient réfléchir en même temps qu’ils jouaient et c'était plus compliqué pour eux. Chaque situation devait traduire une sorte de trame invisible pour eux, mais qui permettrait de raccrocher ensuite chaque situation à une autre. L'enjeu était donc de suivre la trame jusqu’à la partie finale.

Yoan, I'homme capuchonné du film, est un personnage assez intéressant en tant qu'il est distingué des autres membres du groupe de plusieurs façons: assez silencieux, il semble ne pas vraiment appartenir au groupe, mais en être une sorte de satellite. De plus, il regarde à plusieurs reprises frontalement la caméra et rompt ainsi avec le régime de l'observation pour le spectateur.

Ce qui est révélé de Yoan Mudry correspond en partie à sa personne et ce que je connais de lui. De par son parcours plutôt post-punk, émergent encore certaines attitudes de défi comme celle de regarder la caméra. Dans le même temps, Yoan a été mon étudiant lorsque j'enseignais à la HEAD en peinture. Pendant un mois, j’ai pensé que c'était une fille avant de voir dans les dossiers qu'en fait j’avais été piégé par des traits androgynes dont il jouait beaucoup à l'époque. Et c'est l'ambiguïté de ce personnage qui lui procure sans doute cette faculté d'interpellation.

De plus, tout comme Camille Poltera et Marion, Yoan ne fait pas partie du groupe principal qui s'appelle les Marbriers. Les Marbriers étant un groupe assez clanique et fermé, il m’est apparu nécessaire de créer un clivage ou un décalage en y introduisant des personnages solitaires afin de pouvoir filmer ce groupe ainsi reconstitué. De même, le personnage d'Alan n'est pas présent dans le premier tournage. Il a été ajouté pour équilibrer les différences de jeu et cela fonctionne très bien. Pour en revenir à Yoan, j’ai le sentiment qu'il agit comme une sorte de personnage de référence pour le spectateur, celui auquel il est le plus facile de s'identifier.

En connaissant un peu cette scène artistique, on peut constater un important écart entre la place que prennent les revendications politiques dans l'activité de ce groupe et leur absence dans le film. Pourquoi avoir choisi d'effacer ce type d'engagement, ou de ne l'évoquer que par ellipse?

Ce film n'a pas comme sujet les Marbriers ou l'espace d'art qu'ils ont ouvert durant les tournages. Ils ont été choisis car ils sont comme une sorte de groupe spécifique au sein d'une génération qui l'est aussi. Notamment vis-à-vis de la nôtre. Je ne crois pas que leur engagement ait été effacé dans le film. Au contraire, je pense qu'il emprunte une autre voie et donne à voir les choses de manière plus globale et plus souterraine en même temps. Ce qui a été effacé dans le film ce sont les marqueurs géographiques situant l'action à Genève. Pour un non-Genevois, il est très compliqué de reconnaître cette ville. Pourtant, ceux qui en connaissent les lieux nocturnes la reconnaîtront au premier coup d'œil. Ces lieux ont été ouverts durant la période squat de Genève par la génération de leurs 

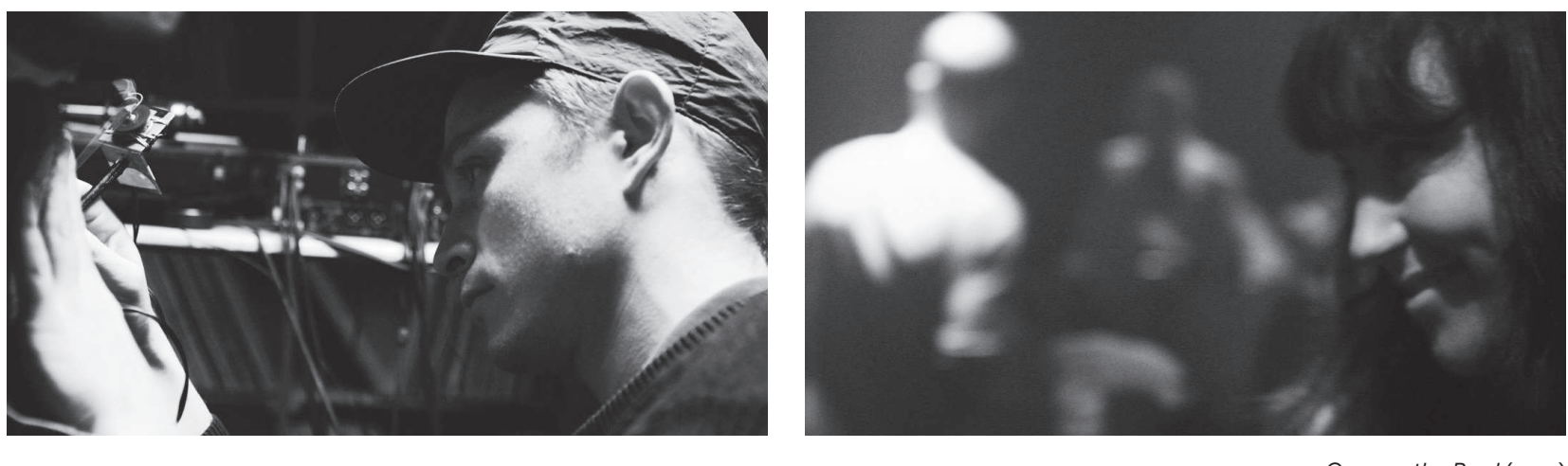

Occupy the Pool (2015)

parents ou des proches de leurs parents. Le film - même s'il ne l'aborde pas directement - parle aussi de ce passage générationnel et des cloisons de plus en plus hermétiques entre les différentes classes d'âge. Lorsque les protagonistes font la fête, ils s'invitent dans des lieux post-squat pour se les accaparer euxmêmes ou s'intégrer à ce legs.

L'aspect «aquarium» et distant de la première partie du film, produit notamment par la teneur flottante des dialogues et la manière de cadrer, est une sorte d'inversion de la seconde partie. Au-delà de l'aspect politique qui n'est pas l'aspect majeur de ce projet, ce sont surtout les symptômes que j’ai cherché à cerner. Pourquoi cette génération est-elle si déconnectée des autres? Pourquoi se sent-elle apparemment minorisée ou non respectée? Quelle est sa place dans l'histoire et surtout quel sens a cette histoire? Quelle est la responsabilité des personnes de la génération au-dessus de la mienne? Ces questions sont propres au monde occidental et se traduisent dans le film par un groupe abandonné ou isolé des autres générations, contraint d'emprunter les coulisses d'un monde dans lequel il ne semble pas avoir de place.
Au-delà de l'aspect purement politique, je crois qu'il y a là surtout une réflexion à avoir sur ce qu'est le réel pour eux, puisque leurs références directes sont avant tout les autres, qui à la fois les protègent et font office de bouclier social. Mais cette notion est ambivalente car le film est construit à partir du réel et sa structuration fait douter de cette notion. Est-ce le réel qui est donné à voir ou le réel est-il situé du côté du spectateur? Cette ambivalence, on la retrouve du côté du jeu. Avec quoi jouent-ils le plus souvent? Certes des accessoires assez futiles, mais aussi les utopies que leur ont laissées les générations précédentes. Finalement ce qu'on observe durant le film c'est aussi cette lutte, comment faire pour que ces utopies ne deviennent pas dystopiques, comment faire pour écrire un récit commun au-delà du legs des années i960, dans lequel ils semblent ne pas se reconnaître.

Le film développe un discours autour de la propriété, que ce soit à travers la possession d'objets - le briquet et la cigarette -, ou par le fait d'occuper une propriété privée, une piscine, sans pour autant chercher à la rendre publique. Cela diffère assez radicalement des mouvements d'occupation des espaces publics, comme "Occupy Wall 
Street», et d'autres mouvements activistes plus récents. Te positionnes-tu par rapport à cela - ne serait-ce que par le titre qui est un syntagme connoté?

Oui, c'est juste. La première écriture date de 20I2, au plus haut des mouvements «Occupy». Ce qui m’a frappé en observant ces mouvements, c'est qu'ils se fédéraient par leur aspect fourre-tout et l'absence de revendications claires. Comme s'il fallait le faire sans savoir pourquoi. Le cadre premier de ce projet était d'ailleurs la ville de Detroit vue depuis son vis-à-vis situé de l'autre côté du pont, côté canadien. Il y avait alors deux mouvements "Occupy» aux revendications très disparates et, oserais-je dire, opaques. Dans le même temps, il y eut aussi un mouvement «Occupy» en Alabama, je crois, où certains participants portaient une arme. Mais le mouvement qui attira mon attention fut probablement le mouvement «Occupy» de Genève où un participant mourut de froid. Je pense que cette idée me marqua, car à ce moment-là j’avais l'intime conviction que ces mouvements ne portaient qu'une revendication, à savoir d'être ensemble et de renouer avec un besoin communautaire minimal. C'est à ce moment que le film a été conçu comme une sorte de naufrage. La seconde écriture du scénario lui a simplement ajouté une préquelle: les fêtes.

Quant à la question de la propriété, c'est très présent à la fois dans le film et dans ce groupe en particulier. Par exemple, avoir un paquet de cigarettes dans ce groupe fait que celles-ci deviennent toutes propriété des personnes présentes (tout comme l'argent souvent). C'est à ce moment-là que j’ai remarqué que les filles étaient plus malignes et cachaient une partie de leurs cigarettes, comme le fait le personnage de Mélanie. Pourtant, le groupe ne produit rien de luimême. Les ressources sont captées en dehors puis partagées et consommées à l'intérieur de ce groupe. C'est en partie cela qui crée une frontière, un inté- rieur et un extérieur. Nous sommes là face à une économie de subsistance étrange, d'autant que presque tous proviennent de la classe moyenne. Ce sont dans ces observations-là que se loge l'aspect politique de ce film. L'ironie du titre est adressée à ceux qui s'attendent à un discours politique lourd; le titre joue avec les attentes. Le film donne à voir de petites actions et des procédures très localisées. C'est le type de quotidien de cette classe d'âge à ce moment précis de leur histoire, et c'est aussi ma manière de renvoyer aux autres générations un modèle qu'elles ne semblent pas désireuses de prendre en compte.

A cet égard, il y a une sorte de violence morale (ou symbolique) que s'inflige ce groupe qui semble chercher à compenser le côté insignifiant du fait d'occuper une piscine. Est-ce une façon d'intégrer une violence subie par cette génération, de se l'approprier?

Il faut comprendre que pour ceux qui arrivent après nous, l'esthétique de la contestation n'emprunte pas forcément les mêmes voies que celles prises par les générations précédentes. Les petites opérations et procédures mises en avant durant le film, qu'elles soient écrites ou réelles, ne sont pas «insignifiantes» pour les protagonistes du film à ce moment de leur histoire. Elles sont une manière - parfois assez violente - de communiquer avec leur entourage mais aussi d'affirmer un modèle qui leur soit propre. La piscine se trouve en droite ligne de cette approche. A aucun moment elle n'est décrite comme un symbole bourgeois, mais comme la phase terminale et communiante d'un périple somme toute assez monotone. Les protagonistes du film s'y retrouvent baignant dans un flux commun. La piscine constitue aussi cette ligne d'horizon confisquée durant tout le film puisque cette ligne était alors représentée par les épaules et têtes des personnes du groupe. 
Il y a cependant quelque chose de l'ordre de la violence qui suinte durant tout le film. Je pense que cela provient du réel. L'approche consistant à partir du réel pour l'écriture du film y est à mon sens pour beaucoup.

Je donne un exemple. Ce groupe aimait s'inviter dans des lieux squattés et c'est ce que raconte la seconde nuit de fête. A ce moment-là, Timothée Endt ne parvenait pas à défoncer la porte d'entrée de manière crédible. Etrangement, c'est un des responsables du lieu qui a résolu le problème en bricolant la porte. Avec cette scène, nous sommes réellement sur un plan symbolique à de nombreux niveaux: dans ce passage d'une génération à une autre où l'échange ne prend pas, dans le caractère très pathétique de la communication entre deux âges (autour d'une porte en plus) qui manifeste le fait que ces deux "générations» ne savent plus se parler. A la suite du premier visionnage de la version finale, la réaction d'amis plus vieux que moi me frappa. Une de leurs questions correspondait à peu près à: «Mais ils sont comme nous à leur âge?», qui me confirma une vraie rupture entre les générations antérieures et celle représentée dans le film. A ce moment-là, ma réflexion fut plutôt de me demander si le film filmait les protagonistes dans une bulle, ou si finalement ce n'était pas plutôt le spectateur qui était dans une bulle. Le réel est certainement le principal enjeu de ce film.

Tu choisis principalement des acteurs qui sont inscrits dans une scène artistique localisable et pourtant cela n'apparaît pas dans le film. Au contraire, même s'ils sont relativement marginaux, les personnages ont des préoccupations et des activités qui forment le quotidien commun à ce milieu social: pourrait-on dire que ton film travaille le genre du teen movie?

Ce n'est pas vraiment la caractéristique principale du film selon moi. S’il y a bien un rapport au genre du teen movie, il provient surtout du fait que je suis issu d'une génération qui s'est trouvée au centre des films de Gus van Sant, Larry Clark ou Harmony Korine. Mais, dans mon esprit, le style Gus van Sant est passé et nécessite une réécriture, un peu comme le dernier film de Korine opère une réécriture de ses premières productions.

Je pense qu'Occupy the Pool emprunte le genre du teen movie, mais par la marge. Le réel me semble nettement plus prégnant que l’aspect générationnel. Il faut comprendre que ce que décrit en partie Occupy the Pool, c'est que nous vivons dans des bulles sociales en partie pour nous protéger du réel. Il y a une logique dès lors à se filmer nous-mêmes, à produire notre propre récit.

Pourrais-tu nous décrire les tensions et les relations entre les valeurs de plans et leurs implications sur le mode de production du film?

En termes d'images, le film est redevable à Gabriel Lobos, le chef opérateur, et Manon Vila, la monteuse. Avec Gabriel, nous sommes partis des visages et en avons fait une norme pour le reste du film. Nous avons été aidés en cela par le choix de la caméra sur le second tournage, une caméra qui donnait un bon rendu des visages et des textures de peau. Cela contribue à donner un effet flou à l'arrière-plan, à participer de cet effet de bulle. Pour la partie sèche (hors de la piscine), nous avons un peu élargi la zone à filmer en l'étendant de la tête à la taille, afin de voir les objets et transactions. L’omniprésence des visages, le peu de plans d'ensemble, la structure immergée dans la piscine pour la captation, confèrent cette force à l'image et son caractère parfois un peu inquiétant. Cet effet d'étrangeté est d'autant plus fort que nous cherchions à effacer les repères géographiques environnants. 
La conséquence pour l'équipe fut d'être réellement immergée dans les situations de tournage. Je parle de «situations de tournage» car nous avions choisi d'organiser de vraies fêtes afin de tourner. Cela a été éprouvant et nous avons dû beaucoup anticiper.

Le tournage a sinon été abordé de manière relativement classique, si ce n'est que l'équipe était relativement réduite et s'apparentait plutôt à ce qui se pratique pour le documentaire. A la fin du tournage, nous disposions d'images conçues pour être montées de manière classique.

Manon Vila, qui vient de la vidéo d'art plus que du cinéma, a débuté par le montage de la partie piscine. Cette partie-là ne fut plus touchée ensuite, car la syntaxe et le style employés furent très convaincants. Son apport, notamment en allant rechercher les moments de «flou», furent surprenants et cela nous servit de référence pour le reste du montage. La partie centrale du film repose néanmoins sur un montage plus classique, qui correspond à ce que l'objet final aurait pu être sans l'apport de Manon.

La construction narrative du film présente de nombreuses ellipses et la progression de l'intrigue choisit de ne pas vraiment encadrer le récit: as-tu construit le film en vue de le diffuser dans certains espaces (salles de cinéma classiques, festivals, lieux dits "alternatifs», etc.)?

Le film a clairement été conçu pour les festivals, du moins durant la première année. Nous étions conscients que ce type de production ne s'adresserait qu'à peu de monde. Nous ne savions pas en revanche pour quel type de spectateur. Il s'avère qu'à ce stade, l'accueil est meilleur du côté du cinéma que de l'art contemporain. Je pense que les problématiques soulevées sont complètement de ce côté et non de l'art qui développe d'autres types de préoccupations.

\section{Lesquelles?}

Il m'est difficile de répondre tant aujourd'hui la situation en art contemporain est floue. En discutant avec de très jeunes étudiants, ceux-ci me répondent avec un grand sourire que l'art contemporain ne les intéresse pas. Je pense, à nouveau, qu'il s'agit d'un rapport au réel, avec l'envie de s'y confronter, de l'apprivoiser. Du point de vue des jeunes artistes, j’ai le sentiment que le cinéma les rapproche du réel tandis que l'art contemporain les en éloigne. Il y a aussi plus de solidarité dans les processus du cinéma que dans ceux de l'art contemporain qui s'avèrent souvent cyniques et excluants. 